\title{
Diamond Window Technology for Electron Cyclotron Heating and Current Drive: State of the Art
}

\author{
Gaetano Aiello, ${ }^{a *}$ Theo Scherer, ${ }^{a}$ Konstantinos Avramidis, ${ }^{\mathrm{b}}$ Natalia Casal, ${ }^{\mathrm{c}}$ Thomas Franke, ${ }^{\mathrm{d} e}$ \\ Mario Gagliardi, Gerd Gantenbein, ${ }^{\mathrm{b}}$ Mark Henderson, ${ }^{\mathrm{c}}$ John Jelonnek, ${ }^{\mathrm{b}}$ Andreas Meier, ${ }^{\mathrm{a}}$ \\ Gabriella Saibene, ${ }^{\mathrm{f}}$ Sabine Schreck, ${ }^{\mathrm{a}}$ Dirk Strauss, ${ }^{\mathrm{a}}$ Manfred Thumm, ${ }^{\mathrm{b}}$ Minh Quang Tran, ${ }^{\mathrm{g}}$ \\ Christoph Wild, and Eckhard Woerner ${ }^{\mathrm{h}}$ \\ ${ }^{a}$ Karlsruhe Institute of Technology, Institute for Applied Materials, Eggenstein-Leopoldshafen, Germany, 76344 \\ ${ }^{b}$ Karlsruhe Institute of Technology, Institute of Pulsed Power and Microwave Technology, Eggenstein- \\ Leopoldshafen, Germany, 76344 \\ ${ }^{c}$ ITER Organization, St Paul Lez Durance, France, 13067 \\ ${ }^{d}$ EUROfusion Consortium, Garching, Germany, 85748 \\ ${ }^{e}$ Max-Planck-Institut für Plasmaphysik, Garching, Germany, 85748 \\ ${ }^{f}$ F4E Joint Undertaking, Barcelona, Spain, 08019 \\ ${ }^{g}$ Swiss Plasma Center, EPFL, 1015 Lausanne, Switzerland \\ ${ }^{h}$ Diamond Materials GmbH, Freiburg, Germany, 79108
}

\begin{abstract}
Nuclear fusion power plants require electron cyclotron (EC) heating and current drive $(H \& C D)$ systems for plasma heating and stabilization. High power microwave beams between 1 and $2 \mathrm{MW}$ generated by gyrotrons propagate in a dedicated waveguide transmission system to reach the plasma at specific locations. Key components in this transmission system are the chemical vapor deposition diamond windows on both the torus and gyrotron sides of the reactor as they allow transmission of high power beams while acting as confinement and/or vacuum boundaries. Diamond windows consist of a polycrystalline diamond disk integrated in a metallic housing. In the conventional configuration, there is one disk perpendicular to the beam propagation direction. A steering mechanism is then used to deploy the fixed frequency beam at different locations in the plasma. This is, for instance, the configuration used in the ITER EC H\&CD system. Movable parts close to the plasma will be problematic for the lifetime of launchers in future fusion reactors like the DEMOnstration nuclear fusion reactor (DEMO) because of the higher heat loads and neutron fluxes. Therefore, one of the alternative concepts is to deploy the beams directly at the desired resonant magnetic flux surface by frequency tuning gyrotrons. In this case, diamond windows able to work in a given frequency range, like the diamond Brewster angle window, are required. It is an elegant and compact broadband window solution with the disk inclined at the Brewster angle with respect to the beam direction. This paper shows the development and the current state of different diamond window concepts including the design, the numerical analyses, and application of standard construction nuclear codes and of a specific qualification program.
\end{abstract}

Keywords - Electron cyclotron system, diamond window, Brewster window, loss tangent, FEM analyses.

Note - Some figures may be in color only in the electronic version.

*E mail: gaetano.aiello@kit.edu 


\section{INTRODUCTION AND BACKGROUND}

Chemical vapor deposition (CVD) diamond windows ${ }^{1}$ are subcomponents of electron cyclotron (EC) heating and current drive $(\mathrm{H} \& \mathrm{CD})$ systems used in tokamaks for a diverse range of applications including plasma heating and control of plasma magnetohydrodynamic instabilities such as sawtooth and neoclassical tearing modes (NTMs). An EC system ${ }^{2}$ consists of gyrotrons, which are the sources for the high-power microwave beams, associated power supplies, transmission lines (TLs), and launchers injecting the beams into the plasma.

To meet the several requirements, the EC launchers shall be capable of depositing the EC power at specific positions inside the plasma. The conventional way consists of sending fixed frequency beams by moveable mirrors to deposit the EC power at defined magnetic flux surfaces in the plasma. This is used, for instance, in ITER for an operating frequency of $170 \mathrm{GHz}$ and a radiofrequency (rf) output power between 1 and $1.5 \mathrm{MW}$ (Ref. 3). The diamond disks of the vacuum windows are perpendicular to the beam direction, and in the case of the ITER torus window, they have a thickness of $1.11 \mathrm{~mm}$ corresponding to three times $\lambda / 2$ (the half wavelength) of $170-\mathrm{GHz}$ rf inside the diamond material. The corresponding gyrotron windows have a disk thickness of $1.85 \mathrm{~mm}$ corresponding to five times $\lambda / 2$. Since both the torus window and the gyrotron window are integer multiples of half the wavelength inside the diamond, the window reflections are minimized. Another possibility is to deploy a beam at different frequencies in the plasma. That is the case for the DEMOnstration nuclear fusion reactor (DEMO), as the higher heat load and neutron flux will make it difficult to mechanically move mirrors, which are directly facing the plasma. With respect to the conventional configuration, the EC power cannot be swept across the plasma cross section, but the rf beam is deployed at a fixed launching angle, and frequency tuning allows a different radial EC power deposition.

In this case, broadband window solutions are required for steering the beam to different positions by changing the EC frequency. A specific solution is given by the conventional configuration of the disk associated with gyrotrons designed for multipurpose (multifrequency) operability. Multipurpose gyrotrons produce beams at frequencies corresponding to wavelengths matching the thickness of the disk (e.g., 136/170/204/238 GHz for a window having the same disk thickness as for the ITER gyrotron window), one at each time. A more general broadband solution is the one associated with frequency step-tunable gyrotrons able to switch the operating frequency within seconds and in steps of 2 to $3 \mathrm{GHz}$, thus allowing a faster and finer-tuning of the location of the EC power deposition into the plasma. In particular, the required speed of frequency step-tunability is related to the characteristic time for the rise of plasma instabilities, like NTMs. Following Ref. 4, this time is of the order of $3 \mathrm{~s}$ for ITER and is expected to be larger ( $\sim 10$ to $20 \mathrm{~s}$ ) for DEMO. The speed of gyrotron frequency step-tunability depends on the speed of change of the magnetic field. For example, at a field of 5 to $6 \mathrm{~T}$, a 2- to 3-GHz step appears to be feasible in $\sim 0.5 \mathrm{~s}$ (Ref. 5). One possible solution for frequency step-tunability is the CVD diamond disk Brewster-angle window, ${ }^{6}$ for which the diamond disk is inclined at the Brewster angle of $67.2 \mathrm{deg}$. Assuming a linear beam for which the polarization direction (direction of the electric field) is in the plane of incidence, the rf power is transmitted for all possible frequencies without any reflection. In reality, assuming a nonideal beam and beam polarization, there is always a small reflection depending on the $\mathrm{rf}$ beam mode. As a consequence, the thickness of the disk is also calculated taking into account a minimum reflection and the necessary structural stability. It should also be noted here that the Brewster window solution, albeit much simpler than others (e.g., paired, mechanically tunable windows), introduces the complication of having to place the polarizers after the window, i.e., in the primary vacuum of the tokamak. This issue, however, is a question to the EC launcher design, and it is beyond the scope of this paper.

Diamond windows consist of an ultra-low-loss polycrystalline diamond disk brazed to copper cuffs and then integrated in a metallic housing. They are located both at the torus side of the fusion reactors as vacuum and confinement (tritium and other hazardous materials such as radioactive dust) boundaries between the TLs and torus vacuum and at the gyrotron side as a vacuum barrier between the tube vacuum and TLs, while allowing the transmission of high-power microwave beams. The most stringent requirements apply to the torus windows, considering their safety function. With respect to other materials, diamond is the only material that complies with the combined microwave, mechanical, thermal, vacuum, and safety requirements. With particular reference to the EC ITER torus window and the Brewster window concept for DEMO, this paper shows the design of these windows, the numerical analyses of the windows aiming to optimize the design and to investigate different design solutions, the application of the nuclear standard codes, the experimental measurements of the loss tangent $(\tan \delta)$ in the diamond 
disk, and the execution of experimental tests like the pressure test on a diamond window mock-up. The activities ongoing for the two types of diamond windows and the next steps toward feasible working windows are discussed together with the development of a specific qualification program including prototyping activities since the windows cannot be completely covered by the standard codes, like the brazing between disk and copper cuffs.

\section{ITER TORUS DIAMOND WINDOW}

A global view of the EC system at ITER is shown in Fig. 1. There are one equatorial launcher (EL) and four upper launchers (ULs) injecting the rf beams into the plasma. Purely for reasons of convention, the waveguide (WG) lines from the diamond windows back to the gyrotrons are called transmission lines, and they are not part of the primary confinement system. The lines from the windows to the launcher plugs are instead named ex-vessel WGs (EWs) while the ones inside the plugs are called in-vessel WGs, and they are part of the primary confinement system. The EL has a total of $24 \mathrm{EWs}$ at the entry, which are grouped into three sets of eight beams, while each UL has eight EW entries grouped into two sets of four beams (a total of $32 \mathrm{EWs}$ for the four ULs). There is one diamond window for each $\mathrm{EW}$, thus resulting in a total of 56 torus windows for the EC system. The design of the window was developed by the support of Fusion for Energy (F4E), which is the European Fusion Domestic Agency.

In the following paragraphs, it will be shown how the torus window has achieved a sufficient mature design by numerical analyses and applications of codes and standards to start the prototyping and testing activity in view of the
ITER final design review (FDR), scheduled in 2020. Technical specifications are currently under preparation to start the call for tender for the manufacturing of three window prototypes soon. The diamond disk already successfully passed its FDR at the end of 2018. The strategy to perform the diamond disk FDR first was mainly dictated by the need to facilitate the long procurement time for the 56 disks, each of which requires weeks to be manufactured as the growth process is slow (CVD growth rate of 0.1 to $10 \mu \mathrm{m} / \mathrm{h}$ in the microwave plasma reactor).

\section{II.A. Design of the Window}

The current design of the torus diamond window is shown in Fig. 2 with the nomenclature of the parts and the proposed sequence of the joints while Fig. 3 indicates the material of the parts. The design was mainly ruled by the need of

1. a rigid outer frame able to withstand the external loads and avoid any stress propagation toward the sensitive inner parts like the disk

2. a WG system able to prevent the generation of parasitic electromagnetic oscillations in small cavities of the window

3. a cooling system with no direct contact between disk and coolant to remove the EC power absorbed in the disk.

A diamond disk with a thickness of $1.11 \mathrm{~mm}$ and diameter of $75 \mathrm{~mm}$ is brazed to two copper cuffs with embedded channels allowing indirect cooling of the disk. This design choice makes the window safer as the coolant

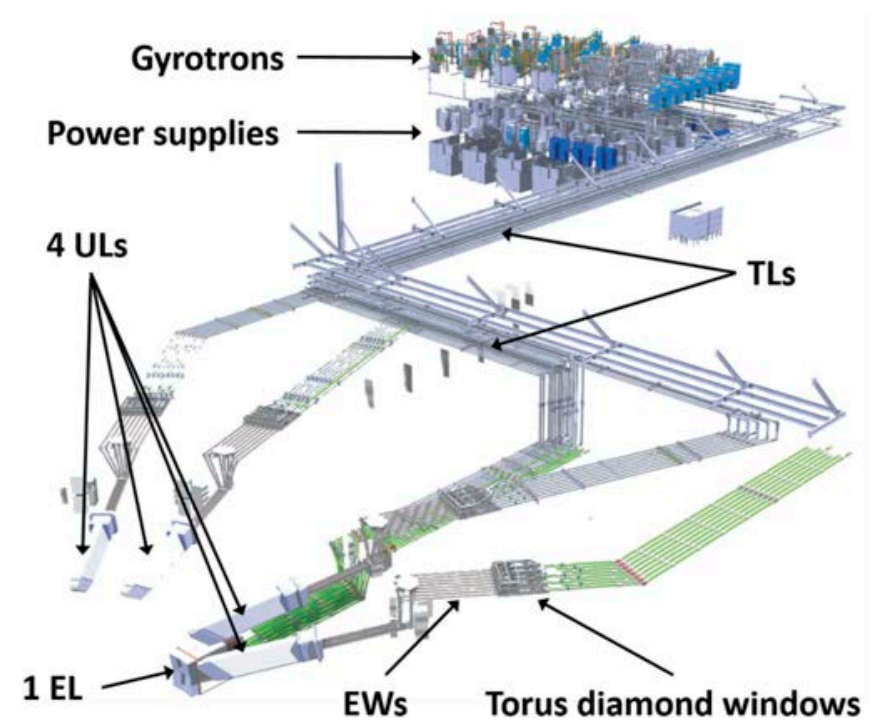

Fig. 1. Electron cyclotron system in ITER.

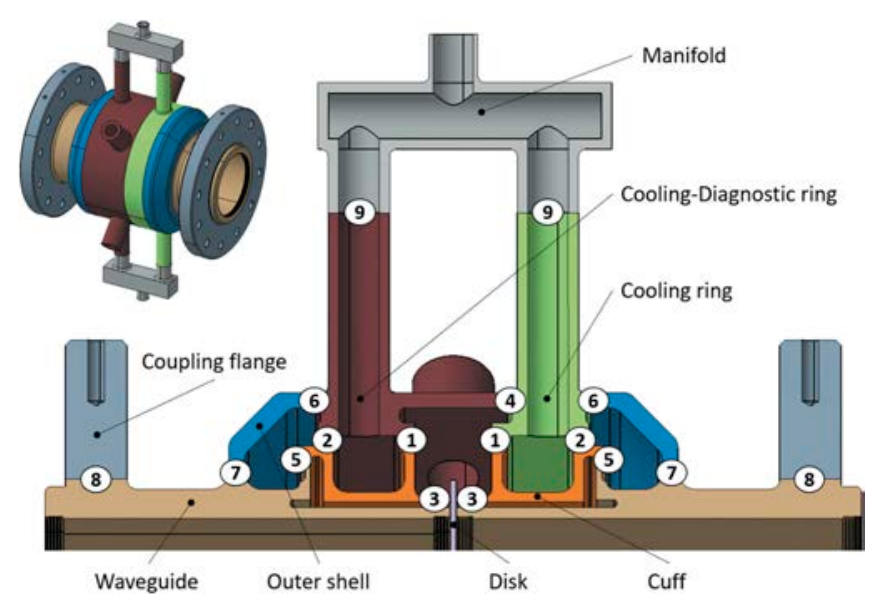

Fig. 2. Current design of the EC torus window unit. The numbers in the circles indicate the proposed sequence of the joints in the unit. 


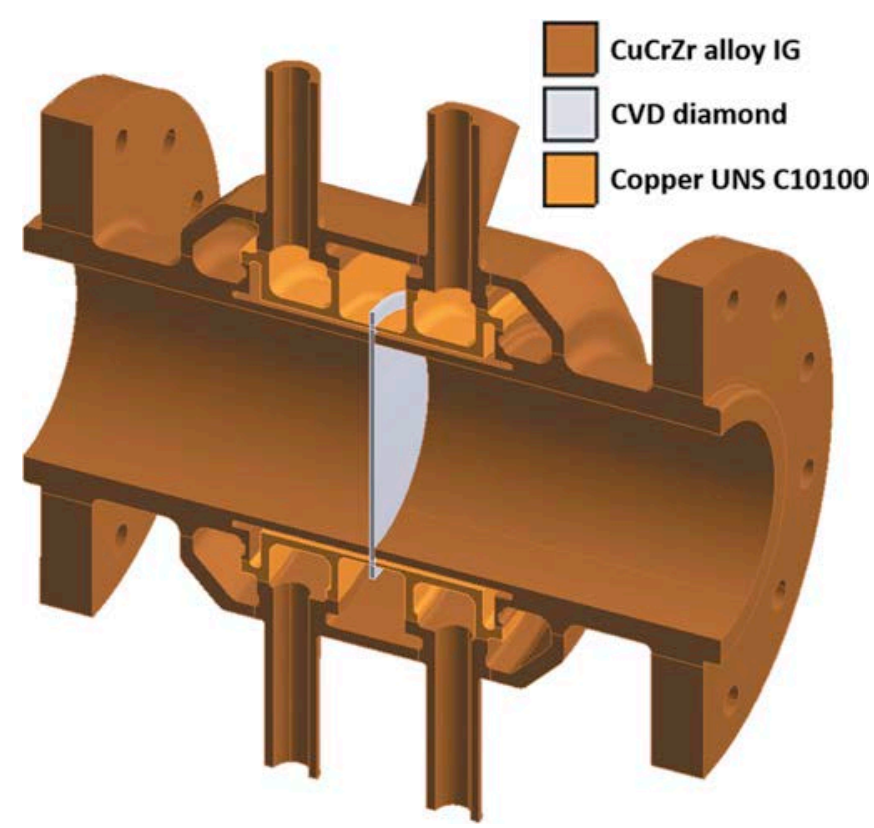

Fig. 3. Materials in the current design of the window unit.

is separated from the disk forming the real barrier. The cuffs are then connected to two circular corrugated WGs of 50-mm inner diameter and made by ITER-grade (IG) copper-chromium-zirconium $(\mathrm{CuCrZr})$ alloy and inserted into the cuffs leaving a $100-\mu \mathrm{m}$ gap with the disk. The cooling channels are closed by the so-called coolingdiagnostic ring and cooling ring also made of $\mathrm{CuCrZr}$. Two CuCrZr outer shells then connect these rings to the WGs thus forming the stiff outer frame of the window. This outer frame acts as an additional confinement barrier, and the real-time monitoring of all interspaces of the window is guaranteed by eight holes located both in the cooling-diagnostic ring and cooling ring. Finally, the WGs are inserted into the EW lines by the $\mathrm{CuCrZr}$ coupling flanges while the $\mathrm{CuCrZr}$ manifolds connect the window to the feeding water circuit. Four diagnostic pipes are located in the cooling-diagnostic ring to connect the window to different diagnostic systems aiming to detect any potential tritium and radioactive dust leakage and any arc generation during the transmission of the $\mathrm{rf}$ waves and also aiming to measure pressure and temperature in the system. The integration of the diagnostics in the window unit is actually under discussion, and different solutions are being taken into account in view of the FDR in 2020.

The brazing is carried out only between the disk and the cuffs while all other parts are joined by electron beam welding except the joints between the pipes of the cooling-diagnostic/cooling rings and the pipes of the manifolds. For these joints, orbital tungsten inert gas welding is foreseen. Looking at Fig. 2, the total number of joints is 19 , in particular 7 × 2 symmetric joints with respect to the middle plane of the disk plus the joint \#4 and the four orbital welds \#9 for the cooling connection (two of them are not visible in Fig. 2). The current design of the window is the result of optimization work by numerical analyses and codes and standards, and it shall be used in the call for tender for the manufacturing of the window prototypes.

\section{II.B. Loss Tangent Measurements}

The measurement of the $\tan \delta$ in the diamond disks aims to check their quality from the microwave transmission perspective. It is a mandatory test for disk acceptance in the qualification program (i.e., the program aiming to check the compliance with the applicable requirements) of the window unit. Very low tan $\delta$ values are required to assure the microwave transmission toward the plasma and consequently low heating of the window. In addition, very low $\tan \delta$ values are sufficient to guarantee that the diamond disk has good mechanical properties, in particular, the ultimate bending strength, of the diamond phase and not of the graphite phase (high values of the tan $\delta$ correlate with a certain amount of graphite phases in CVD diamond). The tan $\delta$ is measured by open spherical and hemispherical Fabry-Perot resonators available in a dedicated measurement facility at Karlsruhe Institute of Technology ${ }^{7}$ (KIT). The spherical setup, consisting of two spherical mirrors and the disk placed at the center, allows high-resolution measurements of the tan $\delta$ at the center of the disk for the bare disk and the disk mounted in the window assembly. The hemispherical setup, consisting of a spherical mirror and a reflecting plane mirror, allows determining the distribution of the tan $\delta$ over the disk surface, and it is possible only for the bare disk. The distribution is then parameterized in terms of the onset (D10), median (D50), and terminal (D90) distribution parameters. These indicate, respectively, the $\tan \delta$ for the $10 \%, 50 \%$, and $90 \%$ fraction of the inspected area, as can be observed in Fig. 4.

In the development of the qualification program of the window unit, acceptance criteria were already defined for the mean D50 and D90 of the $\tan \delta$ distribution at $170 \mathrm{GHz}$ in the bare disks: $3.5 \times 10^{5}$ for D50 and $6.0 \times 10^{5}$ for D90. Diamond disks with the mean D50 and D90 lower than the defined limits shall be accepted for integration into the window assemblies. The $\tan \delta$ measured at the center of the disk is usually used to calculate the EC power absorbed in the disk. This then represents the input to the thermal analysis of the window aiming to calculate the temperature distribution during 

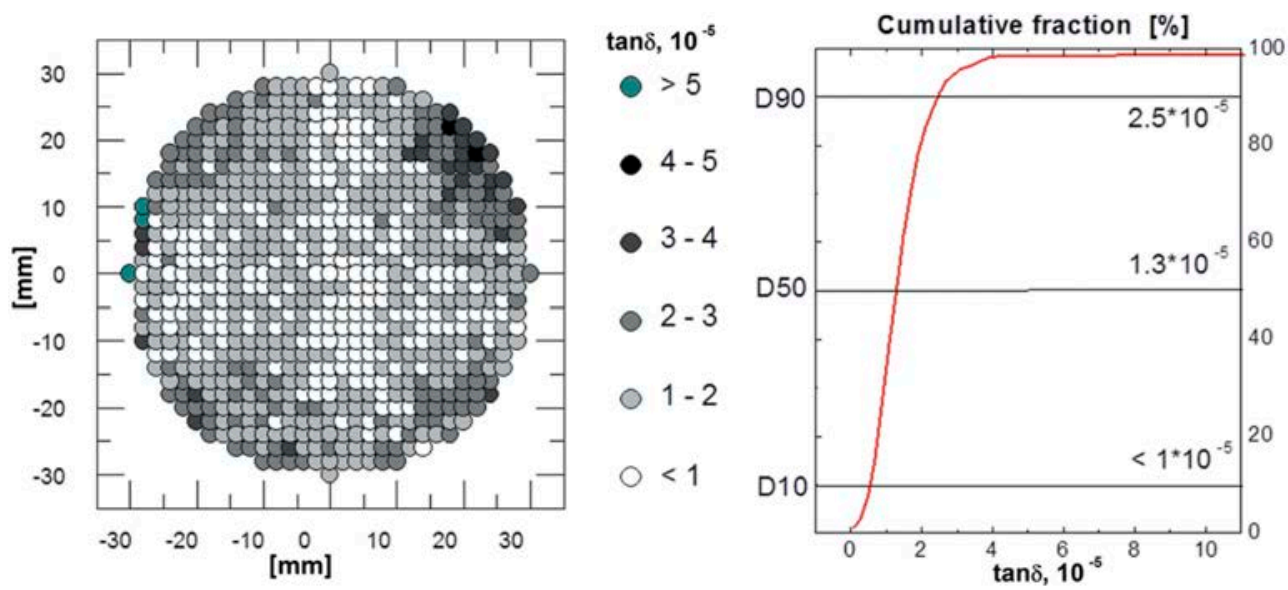

Fig. 4. Loss tangent distribution at $170 \mathrm{GHz}$ for a bare CVD diamond disk manufactured for integration in the window prototype. The mean D50 and D90 meet the acceptance criteria.

normal operation and off-normal events. The absorbed power is calculated according to

$$
P_{a b s}=P_{\text {beam }} \cdot \frac{f}{c} \cdot \pi \cdot\left(1+\varepsilon_{r}\right) \cdot \tan \delta \cdot t
$$

where

$$
\begin{aligned}
P_{a b s} & =\text { absorbed power }(\mathrm{W}) \\
P_{\text {beam }} & =\text { beam power } \\
f & =\text { beam frequency } \\
\varepsilon_{r} & =\text { dielectric constant of diamond } \\
\tan \delta & =\text { loss tangent of diamond } \\
t & =\text { thickness of the disk. }
\end{aligned}
$$

The measurements at the disk center at $170 \mathrm{GHz}$ led to $\tan \delta$ values lower than $2 \times 10^{5}$. Considering $P_{\text {beam }}=1.31 \mathrm{MW}$ at the window location, $f=170 \mathrm{GHz}$, $\varepsilon_{r}=5.67, \tan \delta=2 \times 10^{5}$, and $t=1.11 \mathrm{~mm}$. The EC power absorbed in the disk resulted in only $346 \mathrm{~W}$.

\section{II.C. Numerical Analyses}

The design of the window unit shall meet stringent requirements to guarantee the safety function, the millimeter-wave beam transmission, and the structural integrity during normal operation and off-normal events specified in the ITER load specifications. Several finite element method (FEM) analyses were carried out to check the behavior of the window against the main design drivers and also to optimize the design itself. The analyses may be basically categorized in analyses for the external loads (mainly the severe SL-2 seismic event occurring during the vacuum vessel baking), for the internal loads (mainly the pressure loads acting on the disk), and for the thermostructural loading during the beam transmission, considering also the off-normal event of hot spots. ${ }^{8}$ The hot spots consist of nonaxially symmetric modes in the WGs that lead to excess heating on one side of the WG wall versus the other side. Some analyses, like the ones for the internal loads, have to be considered only as supporting analyses to the experimental tests that actually qualify the window unit. In fact, the window is a unique component as it contains a brittle material (the diamond disk) arranged in a metallic structure requiring a nonstandard industrial joining technique for the integration (brazing between brittle material and ductile material). Once the manufacturing feasibility by the prototyping activity is checked, a final iteration of analyses for the diamond window design shall be performed in view of the FDR scheduled in 2020 .

Figure 5 shows that the analyses ${ }^{9}$ for the design driver related to the external loads acting on the unit led to an advanced optimum design with resulting stresses well below the allowable limits and to the important conclusion that no stress propagation occurs toward the inner sensitive parts of the window, i.e., the disk and the cuffs. The thermal analysis of the window unit during normal operation was run by applying the absorbed power in the disk (see Sec. II.B) in accordance with the power pattern of the $\mathrm{HE}_{11}$ mode beam inside the WGs (Bessel function of order zero) and by applying the heat flux to the inner surface of the WGs. A heat flux of $4071 \mathrm{~W} \mathrm{~m}^{2}$ was applied to the $\mathrm{CuCrZr}$ WGs according to the 1.31-MW design beam. The heat exchange coefficient of $3168 \mathrm{~W} \mathrm{~m}^{2} \mathrm{~K}^{1}$ was applied to the cooling surfaces, consistent with the inlet mass flow rate of $0.0833 \mathrm{~kg} \mathrm{~s}^{1}(5 \mathrm{~L} / \mathrm{min})$ assumed for the complete 

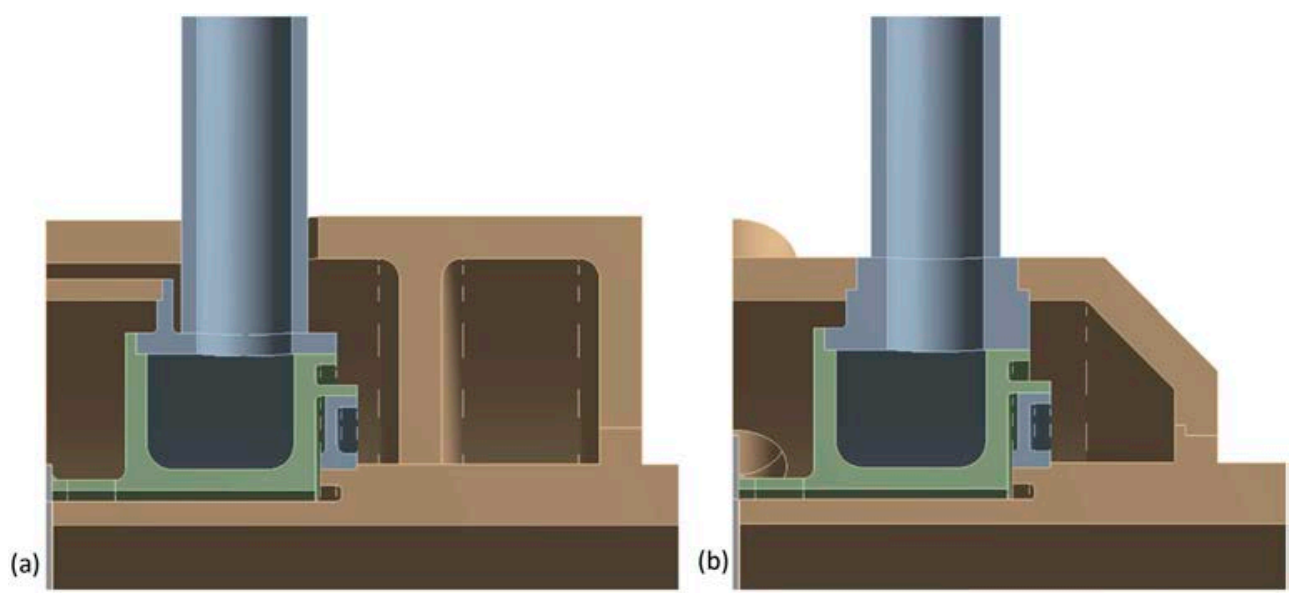

Fig. 5. Evolution of the window unit from initial design (a) toward the compact and optimum design and (b) obtained by running dedicated FEM analyses.

window unit. Figure 6 shows the resulting temperature distribution in the unit. A quarter of the unit was used in the analysis for symmetry reasons. As expected, the maximum temperature is located at the center of the disk, and it amounts to about $95^{\circ} \mathrm{C}$, which is well below the temperature limit for diamond. In fact, the limit for diamond is $250^{\circ} \mathrm{C}$, as at greater temperatures the dielectric properties of diamond start degrading. In the metallic parts, the temperatures are low (safe limit of $80^{\circ} \mathrm{C}$ for the WGs), and the distribution is quite homogeneous thanks to the high thermal conductivity of the CuCrZr alloy.

In the following structural analysis, the thermal stresses were assessed by linearizing them along proper stress classification lines and comparing the results to the $3 S_{m}$ limit, where $S_{m}$ is the allowable stress intensity (123 $\mathrm{MPa}$ for $\mathrm{CuCrZr}$ in the range $20^{\circ} \mathrm{C}$ to $100^{\circ} \mathrm{C}$ ). The resulting stresses in the metallic parts are significantly lower than the limits.

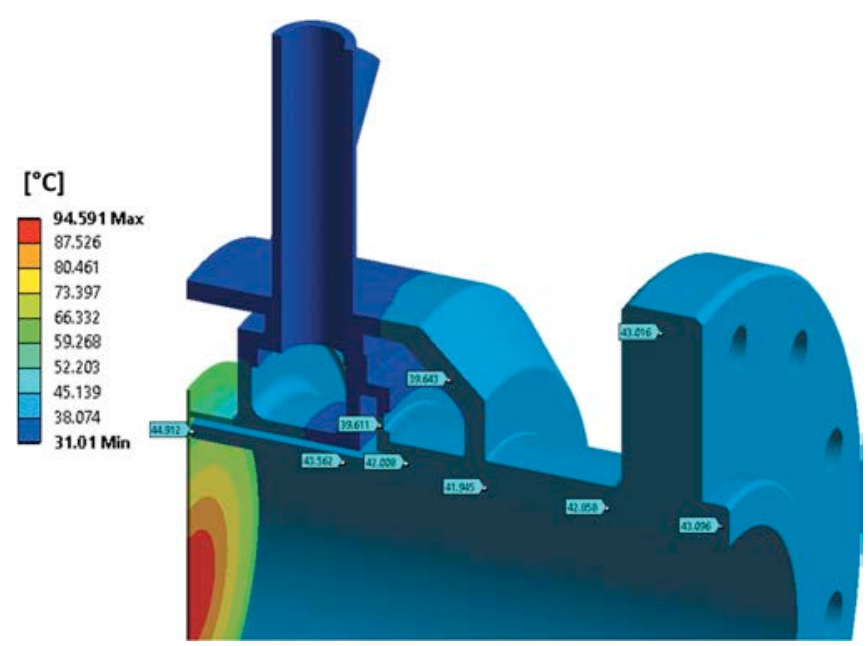

Fig. 6. Temperature distribution in the window unit during normal operation.
In the disk, being a brittle material, the stresses were instead evaluated in terms of first principal stress. The maximum stress in the range 35 to $45 \mathrm{MPa}$ is located in the brazing area, while out of this area, the first principal stress is lower than $22 \mathrm{MPa}$ (a conservative limit stress of $150 \mathrm{MPa}$ is assumed for diamond). Even going to the off-normal event of hot spots, the temperatures are still below the limits, and very high stress safety margins were obtained. It can be concluded that the use of $\mathrm{CuCrZr}$ alloy provides great safety against the uncertainties associated with the thermal loading in the case of a hot spot. In addition, a sensitivity analysis for the cooling of the disk showed that the unit has a very stable thermal performance with regard to variations of the inlet mass flow rate. In fact, when decreasing the flow rate from $20 \mathrm{~L} / \mathrm{min}$ down to $5 \mathrm{~L} / \mathrm{min}$, an increase of only $4^{\circ} \mathrm{C}$ can be observed in the temperature profiles of the disk.

As already mentioned, some analyses are carried out only as supporting computations for the tests in which window mock-ups, prototype windows, and the window units shall be subjected to in the context of the qualification program. For instance, this is the case of the pressure test ${ }^{10}$ at a 2-bar difference across the disk carried out with a window mock-up consisting of the disk brazed to the cupper cuffs. Figure 7 shows the supporting analysis for this test. A two-dimensional analysis was run by applying the 2-bar load on the disk and the cuff, and the resulting maximum principal stress turned out to be of the order of $60 \mathrm{MPa}$ at the critical locations. The 2-bar event is the worst pressure event (an extremely unlikely event) in the vessel corresponding to a multiple in-vessel pipe failure, and it is a required event (ITER safety) to be considered for the window design. The test was successfully carried out, and it contributed to the successful FDR of the 1.11-mm disk. 


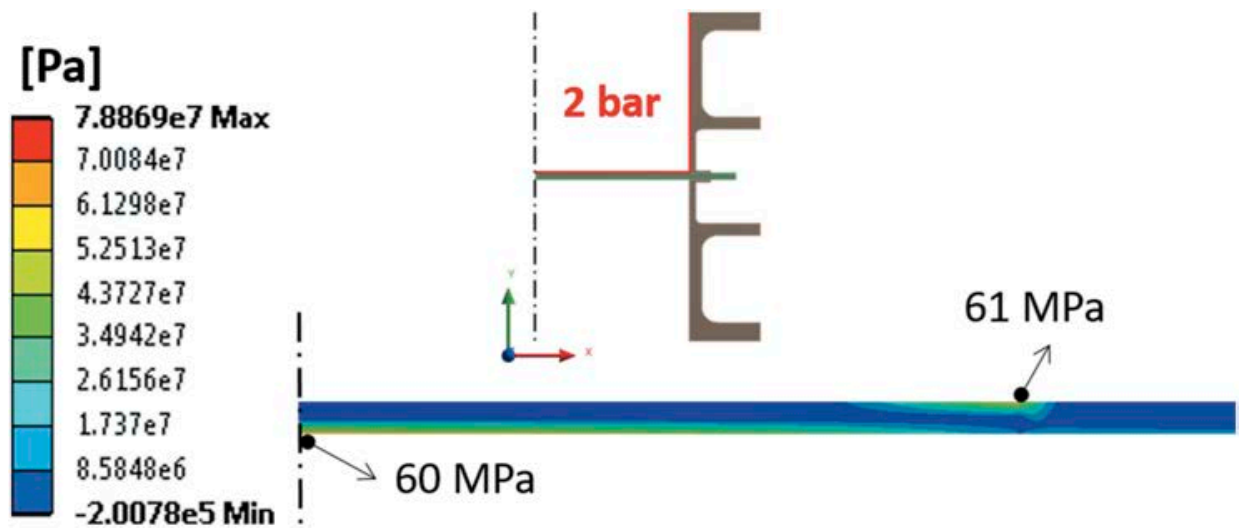

Fig. 7. Supporting analysis for the 2 bar pressure test on the window mock up formed by disk and cuffs.

\section{II.D. Codes and Standards}

The design of the window unit optimized by FEM analyses (Fig. 5b) was further improved ${ }^{11}$ considering the applicable requirements given by ASME Boiler and Pressure Vessel Code (BPVC), Section III, "Rules for Construction of Nuclear Facility Components," Division 1, Subsection NC, "Class 2 Components" (BPVC III-NC). In fact, BPVC III-NC shall be applied to the design, manufacturing, assembling, and qualification of the unit. In particular, the requirements stating that the welded joints in the unit shall have complete joint penetration and full fusion to occur between the pieces (NC-4262a) and also that they shall be fully radiographed (NC-5252) were considered. For instance, as can be observed in Fig. 8, the steps at the location of the joints, originally inserted to help the joining of the several parts, were removed. This allows a complete joint penetration like for instance at the welds between the WGs and the outer shells.

\section{II.E. Qualification Program}

The diamond window unit is actually covered by the applicable BPVC III-NC requirements, but some features like the diamond-copper brazing are out of the standard codes. Therefore, a dedicated qualification program of the unit has to be defined, and it is under development. The program includes testing and prototyping activities, and it also aims to help define the acceptance criteria for the various tests. This path represents the base for the writing of the final qualification program that shall be used for the series production window units. Functional, design, safety, operational, and quality requirements and requirements related to the loading conditions are being defined for the unit. Each unit in series production shall be considered as qualified when all the applicable requirements shall be met through BPVC III-NC and the final qualification program.

The program defines tests that shall be carried out for the bare disk, for the disk integrated in the window
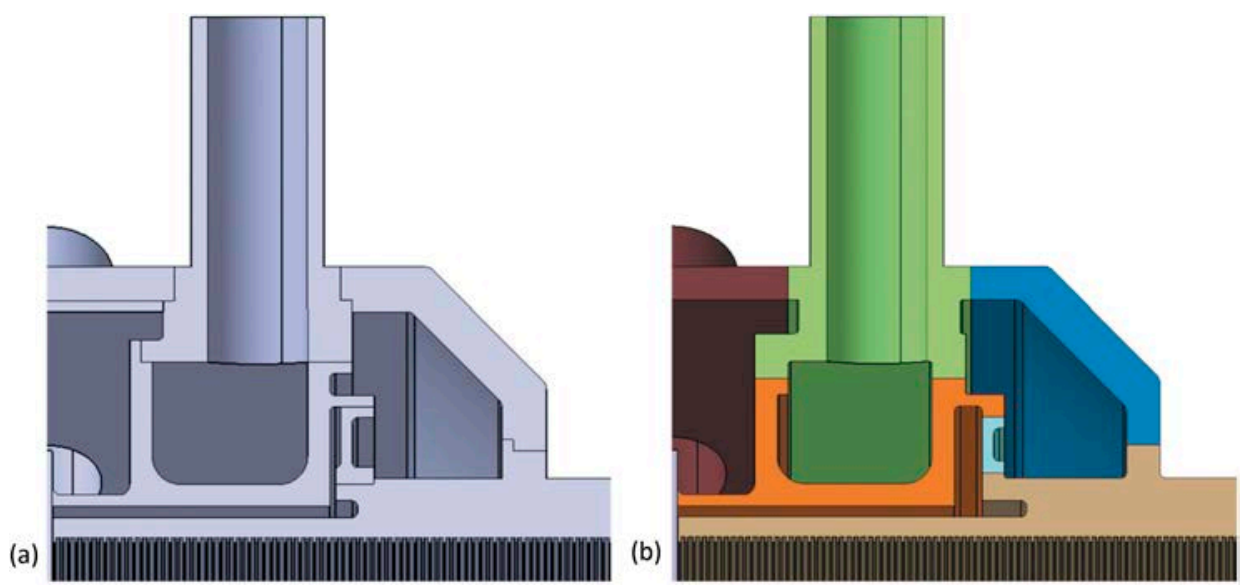

Fig. 8. Evolution of the window unit from (a) the design optimized by FEM analyses toward (b) the design optimized by BPVC III NC. 
subassembly (formed by the disk, cuffs, cooling and cooling-diagnostic rings), and for the complete window. $^{7}$ The tests refer, for instance, to the check of the geometrical tolerances, the leakage rates at the joints, and the $\tan \delta$ measurements. In particular, the latter is important to check the quality of the diamond disk with respect to the microwave transmission and to check that it is not impacted by the brazing and welding steps of the unit. In the context of the testing and prototyping activities, successful pressure tests were already carried out on window mock-ups formed by the disk and the cuffs against the different pressure gradients expected to occur across the disk during the window lifetime. ${ }^{10}$

\section{DEMO BREWSTER-ANGLE DIAMOND WINDOW}

The Brewster-angle diamond window is a key component in the context of the frequency tunable gyrotrons. It consists of an elliptical CVD diamond disk brazed to two copper WGs at the Brewster angle of 67.2 deg for diamond, as shown in Fig. 9. The operation of this window concept was successfully shown for a high-power gyrotron $(\sim 1 \mathrm{MW})$ working in the short-pulse regime $(<10 \mathrm{~ms})$ without any cooling of the window. ${ }^{12}$ In the frame of the DEMO H\&CD Work Package of the Power Plant Physics and Technology program launched by the EUROfusion Consortium, this window concept is being investigated for long-pulse gyrotron operation at 2-MW power levels. Three main issues to solve are identified along this path. The first challenge is to produce a very large-area diamond disk suited for a WG of 63.5-mm inner diameter, compatible with 2-MW microwave transmission. In fact, looking at Fig. 9, it can be observed that with a WG aperture greater than $50 \mathrm{~mm}$, the diamond disks are required to have a diameter greater than $140 \mathrm{~mm}$, which represents the current limit for optical-grade CVD diamond growth.

An aperture of $63.5 \mathrm{~mm}$ requires a minimum disk diameter of $180 \mathrm{~mm}$ at the Brewster configuration and about 2-mm thickness. Available state-of-the-art microwave plasma reactors are not capable of growing diamond disks of such size. In collaboration with the industrial partner Diamond Materials, ${ }^{\text {a }}$ experiments with an unconventional method were thus carried out on a small scale aiming to join diamond fragments by overgrowing the joint gap with diamond to obtain large diamond disks. The hot filament method $^{13}$ was applied, and a dedicated reactor was designed, assembled, and tested to grow two diamond plates together. Before joining the two plates shown in Fig. 10 with the diamond overgrowth, several diamond deposition tests were performed to optimize the parameters of the hot filament method. For instance, variations between the tests included the use of different diameters of tungsten filament and different substrate temperatures, gas flows, and reaction pressures. A strong dependence of the growth rate and the quality of the diamond crystals was found with respect to the distance of the filament. The best growth with respect to quality and growth rate was observed within a distance of less than $3 \mathrm{~mm}$. However, when the filament is too close to the substrate (less than $1 \mathrm{~mm}$ ), the material quality drastically drops as atoms of the filament are incorporated into the diamond lattice (typically tungsten). Using the optimized parameters, the overgrowth of the two plates was carried out for $150 \mathrm{~h}$ leading to the positive result that the two plates grew together and formed a solid compound as shown in Fig. 11. However, there are many drawbacks affecting this method like the very low growth rate $(0.1$ to $1 \mu \mathrm{m} / \mathrm{h})$ and the potential poor optical grade as atoms of the filament are anyway incorporated into the diamond lattice and thus the expected high microwave absorption of the hot filament deposited diamond (expected high values of the $\tan \delta$ ).

The conclusion of this investigation was that joining is not the preferable way to go toward large

${ }^{a}$ Diamond Materials GmbH, Hans Bunte Str. 19, 79108 Freiburg, Germany; http://www.diamond materials.com/EN/index.htm.

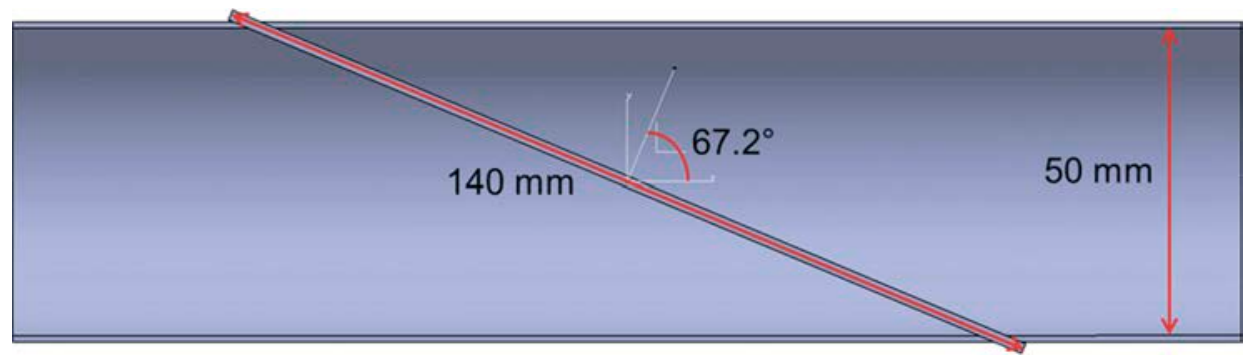

Fig. 9. Diamond Brewster window configuration. 


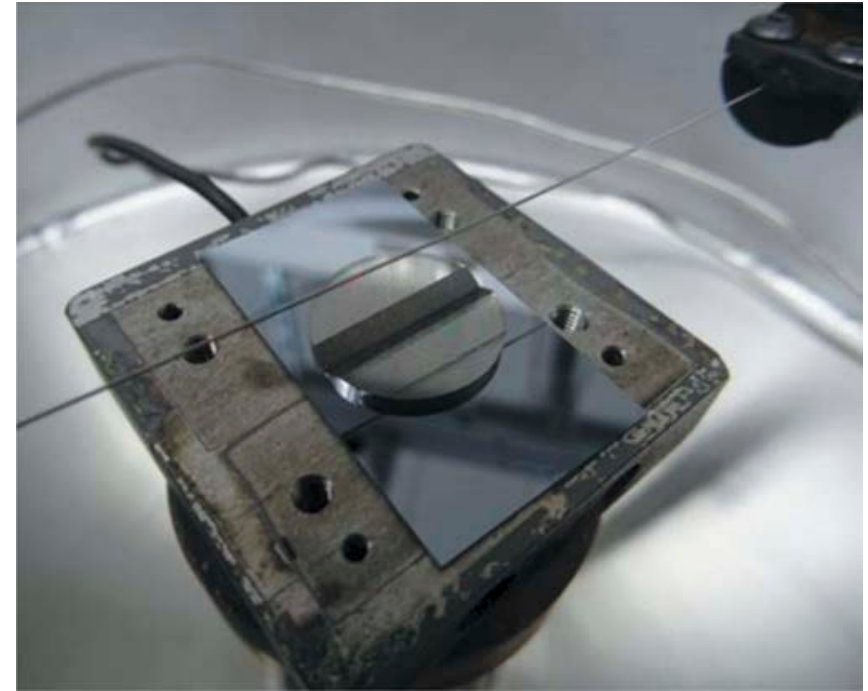

Fig. 10. Diamond plates with the hot filament placed on the joint gap. The position of the filament is not adjusted in height in the picture.

(a)

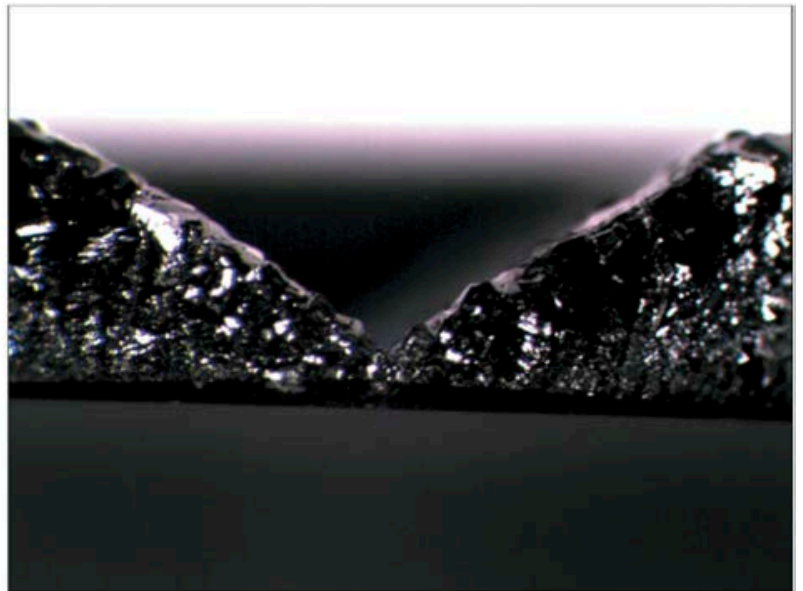

(b)

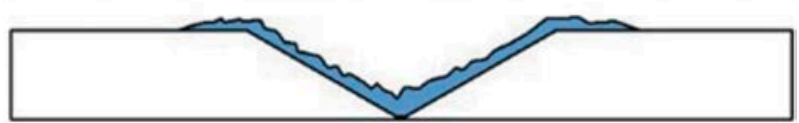

Fig. 11. (a) Solid compound formed by the two dia mond plates overgrown by the hot filament method. The model in (b) better shows the result after $150 \mathrm{~h}$ of deposition.

Brewster-angle diamond disks. The best approach is to directly grow the $180-\mathrm{mm}$ diamond disks in the microwave plasma reactor, and always in collaboration with Diamond Materials, first growth test experiments have already been initiated toward the target of a $180-\mathrm{mm}$ disk with 2-mm thickness. New technologies are thus being investigated as this is a new field for diamond manufacturers. Two growth experiments for parameter optimization were already successfully carried out resulting in rather homogeneous diamond wafers with thickness of only about 300 to $450 \mu \mathrm{m}$. One of these wafers is shown in Fig. 12. Unfortunately, because of the very small thickness, the two wafers did not remain in one piece after dissolving the silicon substrate. However, it was possible to cut a 39-mm-diameter disk from breaking the disks, and it was used for the $\tan \delta$ measurements at KIT. The $\tan \delta$ was measured at the disk center by the spherical Fabry-Perot resonator and was found to be lower than $1 \times 10^{4}$. The target of a $180-\mathrm{mm}$ disk of 2-mm thickness having mechanical properties and $\mathrm{rf}$ quality like the ones of the disks manufactured for the ITER torus window unit is not straightforward, and it requires intense research activity. Several diamond growth experiments are currently ongoing at the laboratories of Diamond Materials in Freiburg to optimize the growth parameters aiming to meet the target.

The second challenge in the Brewster-angle window development toward long-pulse operation is the proper joining of the disk to the WGs via brazing or other techniques able to reduce the manufacturing residual stresses in the window. In fact, compared to the ITER torus window, the configuration in the Brewster window is more complex due to the skewed position of the disk and the consequent asymmetry in the unit. Finally, the last challenge is the design of a cooling layout able to guarantee proper removal of the heat absorbed in the disk during beam transmission. Different conceptual cooling layouts ${ }^{14}$ were already investigated by FEM thermal and structural analyses considering different power and frequency scenarios $(2 \mathrm{MW}$ at $170 \mathrm{GHz}$, $1.5 \mathrm{MW}$ at $240 \mathrm{GHz}$, and $2 \mathrm{MW}$ at $240 \mathrm{GHz}$ ). An example of these layouts is shown in Fig. 13. The

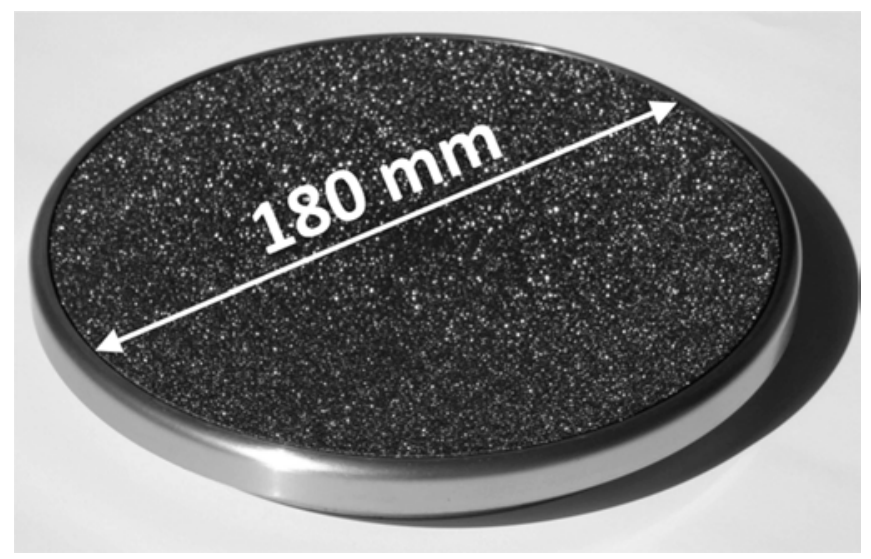

Fig. 12. First diamond disk of $180 \mathrm{~mm}$ with thickness of only about 300 to $450 \mu \mathrm{m}$. The disk as shown in the photograph was still attached to the silicon substrate. 

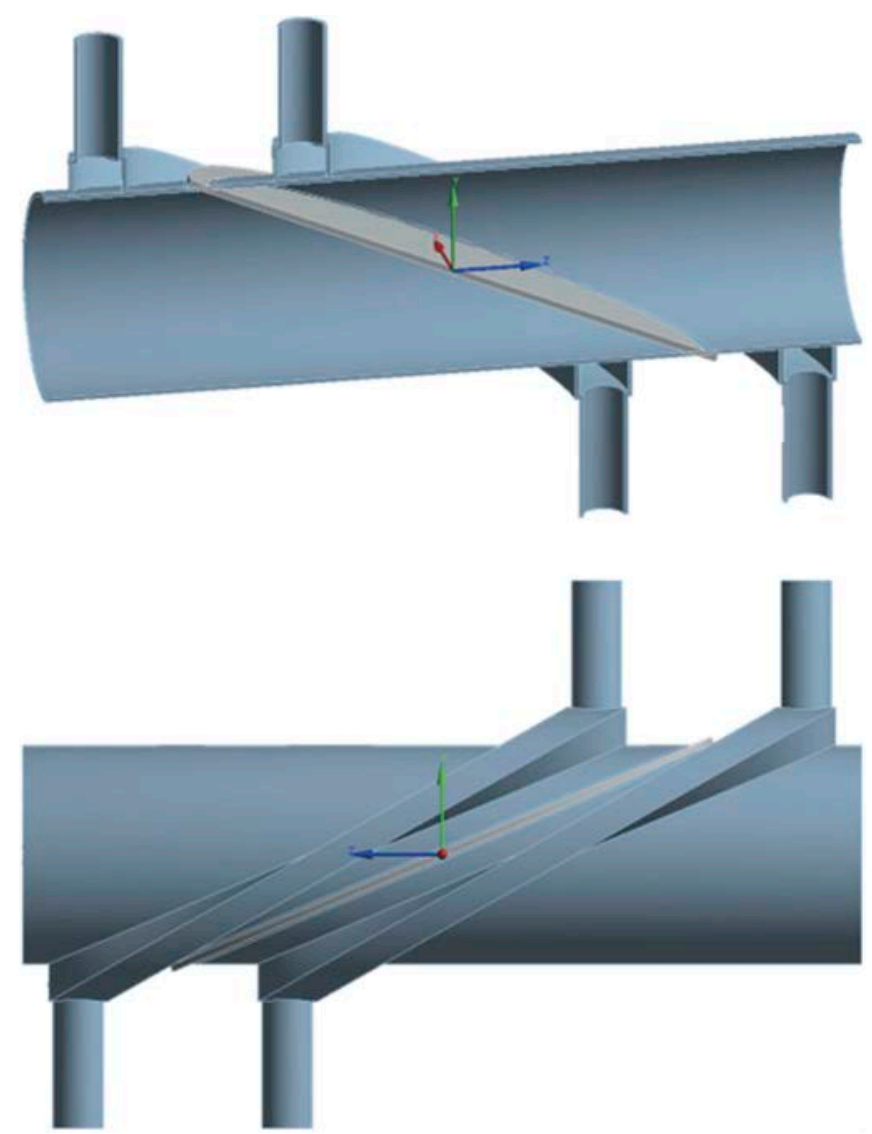

Fig. 13. Conceptual cooling layout in the diamond Brewster window.

analyses were run for a diamond disk having an elliptical shape with major axis of $140 \mathrm{~mm}$, minor axis of $75 \mathrm{~mm}$, and thickness of $1.7 \mathrm{~mm}$. The analyses showed the necessity of having cooling channels that follow the skewed position of the disk; otherwise, the temperatures in the diamond disk due to the millimeterwave losses result in values beyond the temperature limit for diamond $\left(250^{\circ} \mathrm{C}\right)$.

\section{CONCLUSIONS}

In this paper, the development and the current state of the diamond window concepts for the EC H\&CD applications both in ITER and DEMO were discussed. The diamond disk in the ITER torus window already successfully passed the FDR in 2018. The window assembly has already achieved a sufficient mature design to start the prototyping and testing activity in view of the FDR, scheduled in 2020. The manufacturing of window prototypes is essential to check the feasibility of the proposed manufacturing and assembling sequence of the unit. For DEMO, many efforts are addressed to the development of the Brewster-angle diamond window for long-pulse step-tunable gyrotron operation at 2-MW power.

\section{Acknowledgments}

This work was supported by F4E under contract num bers F4E GRT 615 and F4E OPE 467. This work has been also carried out within the framework of the EUROfusion Consortium and has received funding from the Euratom research and training program 20142018 under grant agree ment number 633053. The authors are finally thankful to Diamond Materials for the experiments on the diamond disks.

\section{References}

1. M. THUMM, "Development of Output Windows for High Power Long Pulse Gyrotrons and EC Wave Applications," Int. J. Infrared Milli. Waves, 19, 1, 3 (1998);

2. T. OMORI et al., "Overview of the ITER EC H\&CD System and Its Capabilities," Fusion Eng. Des., 86, 6 8, 951 (2011);

3. D. STRAUSS et al., "Progress of the ECRH Upper Launcher Design for ITER,” Fusion Eng. Des., 89, 7 8, 1669 (2014);

4. O. SAUTER et al., "On the Requirements to Control Neoclassical Tearing Modes in Burning Plasmas," Plasma Phys. Control. Fusion, 52 (2010);

5. Gyrotron magnet suppliers [confidential], personal communi cation, June 2019.

6. X. YANG et al., "A CVD Diamond Disk Brewster Window for a Frequency Step Tunable 1 MW Gyrotron," Int. J. Infrared Milli. Waves, 24, 12, 2003 (2017);

7. S. SCHRECK et al., "ITER ECRH Upper Launcher: Test Plan for Qualification of the Diamond Torus Window Prototype III," Fusion Eng. Des., 109 111, 1232 (2016);

8. J. P. ANDERSON et al., "Heating Effects in Overmoded Corrugated Waveguide for ITER," J. Infrared Milli. Terahz. Waves, 37, 55 (2016); 
9. G. AIELLO et al., "ITER Torus Diamond Window Unit: FEM Analyses and Impact on the Design," IEEE Trans. Plasma Sci., 47, 7, 3289 (2019);

10. S. SCHRECK et al., "Pressure Tests Supporting the Qualification of the ITER EC H\&CD Upper Launcher Diamond Window," Fusion Eng. Des.;

11. G. AIELLO et al., "Design Evolution of the Diamond Window Unit for the ITER EC H\&CD Upper Launcher," Fusion Eng. Des.;
12. G. GANTENBEIN et al., "First Operation of a Step Frequency Tunable 1 MW Gyrotron with a Diamond Brewster Angle Output Window," IEEE Trans. Electron. Devices, 61, 6, 1806 (2014);

13. D. C. HARRIS, Infrared Window and Dome Materials, SPIE, Bellingham, Washington (1992).

14. G. AIELLO et al., "Cooling Concepts for the CVD Diamond Brewster Angle Window," Proc. 42nd Int. Conf. Infrared, Millimeter, and Terahertz Waves (IRMMW THz 2017), Cancun, Mexico, August 27 September 1, 2017, IEEE (2017). 
Karlsruher Institut für Technologie

\section{Repository KITopen}

Dies ist ein Postprint/begutachtetes Manuskript.

\section{Empfohlene Zitierung:}

Aiello, G.; Scherer, T.; Avramidis, K.; Casal, N.; Franke, T.; Gagliardi, M.; Gantenbein, G.; Henderson, M.; Jelonnek, J.; Meier, A.; Saibene, G.; Schreck, S.; Strauss, D.; Thumm, M.; Tran, M. Q.; Wild, C.; Woerner, E. Diamond Window Technology for Electron Cyclotron Heating and Current Drive: State of the Art. 2019. Fusion science and technology, 75 doi: $10.5445 / \mathrm{IR} / 1000099495$

Zitierung der Originalveröffentlichung:

Aiello, G.; Scherer, T.; Avramidis, K.; Casal, N.; Franke, T.; Gagliardi, M.; Gantenbein, G.; Henderson, M.; Jelonnek, J.; Meier, A.; Saibene, G.; Schreck, S.; Strauss, D.; Thumm, M.; Tran, M. Q.; Wild, C.; Woerner, E. Diamond Window Technology for Electron Cyclotron Heating and Current Drive: State of the Art. 2019. Fusion science and technology, 75 (7), 719-729. doi:10.1080/15361055.2019.1643690

\section{Lizenzinformationen: KITopen-Lizenz}

